\title{
EDITORIAL
}

\section{Chlamydia pneumoniae: crossing the barriers?}

\author{
F. Blasi*, S. Centanni", L. Allegra*
}

Chlamydia pneumoniae has been recognised as a cause of respiratory tract infections and implicated as a potential risk factor or causative agent in different extrapulmonary diseases including atherosclerosis, multiple sclerosis, and Alzheimer's disease [1-3]. Being an obligate intracellular bacterium, $C$. pneumoniae has been detected in circulating monocytes and can activate inflammatory processes in epithelial, endothelial and smooth muscle cells in vitro [4].

In the present issue of the European Respiratory Journal $(E R J)$, GIEFFERs et al. [5] report an animal model showing that intratracheal infection with $C$. pneumoniae is followed by systemic dissemination of the infection mediated by peripheral blood mononuclear cells (PBMCs). The authors, on the basis of both the animal model and in vitro study results, hypothesise a "cellular model" for $C$. pneumoniae dissemination. Infection of the lung is characterised by an early phase dominated by granulocytes, and a late phase dominated by alveolar macrophages. Alveolar macrophages, infected by granulocytes, would migrate through the mucosal barrier, using lymphatic tissue, and gain access to the systemic circulation as PBMCs reaching the spleen and the vasculature. The conclusions are mostly inferred on the basis of cell morphology in the absence of definitive determination of infected cell types in extra-pulmonary tissues. Nonetheless, the proposed "cellular model" hypothesis is intriguing and undoubtedly consistent with other recently published studies.

WARK et al. [6] analysed the relationship between airway inflammation and serological response to $C$. pneumoniae in acute severe asthma. At presentation with acute asthma, the sputum total cell count was increased in $C$. pneumoniae antibody responders compared to nonresponders, and $C$. pneumoniae responders had significantly more sputum neutrophils compared to nonresponders. MoAzED et al. [7] demonstrated that monocytes may act as vectors and systemically disseminate C. pneumoniae, and BLASI et al. [8] showed a good correlation between $C$. pneumoniae detection in PBMCs and in atherosclerotic plaques. However, in order to adhere and migrate through the vessel wall, monocytes have to go through a highly coordinated process, which requires the activation of different adhesion receptors in a cascade-like fashion. MAY et al. [9] report that C. pneumoniae infection induces rolling and adhesion of macrophages to the noninflamed vessel wall of noninfected, nonatherosclerotic mice. C. pneumoniae-infected monocytic cells show enhanced transmigration and attach to the endothelium via the activated integrins very late antigen 4 (VLA-4), and the activation of the two $\beta_{2}$-integrins lymphocyte function-associated antigen-1

\footnotetext{
*Institute of Respiratory Diseases, University of Milan, IRCCS Ospedale Maggiore di Milano, and ${ }^{\#}$ Institute of Respiratory Diseases, University of Milan, Respiratory Unit, San Paolo Hospital, Milan, Italy.

Correspondence: F. Blasi, Institute of Respiratory Diseases, University of Milan, Pad. Litta, IRCCS Ospedale Maggiore di Milano, via F. Sforza, 35 I-20122 Milano, Italy. Fax: 39 0250320628. E-mail: francesco.blasi@unimi.it
}

(LFA-1) and macrophage antigen-1 (MAC-1), involving the urokinase receptor (UPAR). This study demonstrates that C. pneumoniae-infected monocytes may be armed to invade noninflamed subendothelium and initiate inflammatory processes. The data indicate that $C$. pneumoniae has the potential to induce a functionally active, adhesive state in monocytic cells by activation of the integrin adhesion receptor system. Therefore, $C$. pneumoniae is not just transported into the subendothelium by monocytes as an innocent bystander, but can actively contribute to the monocyte recruitment to the preferential sites of atherosclerotic lesions. Moreover, this study suggests that $C$. pneumoniae-infected circulating monocytes may have the capacity to induce an adhesive phenotype in adjacent, noninfected monocytes.

Monocytes perhaps also act as the vehicle for trafficking C. pneumoniae across the blood/brain barrier. PBMCs may function as a means by which $C$. pneumoniae enters the central nervous system (CNS) to induce neuroinflammation in Alzheimer's disease and in multiple sclerosis [10]. $C$. pneumoniae infection has been shown to stimulate transendothelial entry of monocytes through human brain endothelial cells (HBMEC). This entry is facilitated by the upregulation of vascular cell adhesion molecule-1 and intercellular adhesion molecule-1 on HBMECs and a corresponding increase of LFA-1, VLA-4, and MAC-1 on monocytes.

An association between $C$. pneumoniae and multiple sclerosis has been proposed, based on the higher frequency of its detection in the cerebrospinal fluid (CSF) of multiple sclerosis patients compared to neurological controls. Multiple sclerosis is an inflammatory demyelinating disease of the CNS of unknown etiology. Current knowledge supports a multifactorial aetiology in which both genetic and environmental factors (including microbial agents) may concur. Interestingly, experimental autoimmune encephalomyelitis (EAE), the experimental animal model of multiple sclerosis, has been successfully induced using a $C$. pneumoniae peptide analogue of rat myelin basic protein [11]. Although multiple sclerosis and EAE are obviously two different entities, this study provides the first indication of a possible direct contribution of $C$. pneumoniae to the pathophysiology of (experimental) demyelination. The presence of $C$. pneumoniae in human CSF does not actually prove that the organism causes or triggers multiple sclerosis: chlamydial infection of the CNS may just represent an opportunistic, secondary event in the disease. Even in this case, however, the presence of the organism may exacerbate/modulate a pre-existing pathogenic process. This is also supported by the finding that $C$. pneumoniae polymerase chain reaction (PCR)-positive patients have more active lesions than $C$. pneumoniae PCR-negative/patients suggesting a role for $C$. pneumoniae in fostering chronic inflammatory stimulation within the CNS [2]. It can be hypothesised that C. pneumoniae might act as cofactor capable of fuelling previously established inflammatory and demyelinating processes and promote a more active disease.

GIEFFERS et al. [5] showed a good correlation between the presence of $C$. pneumoniae in PBMCs both in the lung and in 
the vasculature. These data are consistent with the results reported by BLASI and co-workers [8, 12] who showed that $C$. pneumoniae DNA identification is similar in biopsy specimens (vascular and bronchial) and PBMCs, which suggests that blood PCR may be a useful tool for identifying patients with chronic $C$. pneumoniae infection.

Clinical persistence is probably a key concept in C. pneumoniae infection pathogenesis. Microbial persistence is a state of infection during which the host immune response does not eliminate the pathogen, thereby resulting in continuing damage to the host. Persistent infection may amplify airway inflammation in asthma and chronic obstructive pulmonary disease (COPD), but also in extrapulmonary diseases such as atherosclerosis, multiple sclerosis and Alzheimer's disease.

STEPHENS [13] has recently revised the possible pathogenic mechanisms of $C$. pneumoniae infection. He underlines that C. pneumoniae can induce an inflammatory process elicited by infected host cells that is necessary and sufficient to account for chronic and intense inflammation and the promotion of cellular proliferation, tissue remodelling and scarring, the ultimate causes of disease sequelae.

The cellular responses of epithelial cells, the primary home for $C$. pneumoniae, can be reliably induced upon acute, chronic and persistent infection. The cellular processes of the epithelial cells, elicited by chlamydial infection, cause the influx of inflammatory neutrophils, T-cells, B-cells and macrophages that are stimulated by the pro-inflammatory cytokine and chemokine environment. These cells become activated in both antigen-nonspecific and, for re-infection, antigen-specific responses to produce their own repertoire of cytokines and growth factors. The induction of host cell cytokines will promote foci of inflammatory responses in addition to promoting cellular proliferation, tissue remodelling and healing processes that, if persistent, result in scarring.

The possible role of chronic-persistent infection is suggested in asthmatic children where persistent clinical features are associated with $C$. pneumoniae infection, indicating that this infection should be investigated and treated in case of persistent asthmatic symptoms [14-15]. On the other hand, in adults with asthma, higher titres of antibodies directed against $C$. pneumoniae are associated with more severe clinical disease [16]. The intense neutrophil influx demonstrated in acute severe asthma is potentially a potent source of proteolytic enzymes with the ability to damage and activate the airway epithelium, whereas neutrophil elastase can cause eosinophil degranulation [6]. The inflammatory changes caused by infection with $C$. pneumoniae have the potential to amplify the inflammation and airway damage present in asthma. Furthermore, standard asthma treatment may potentially enhance this response, given the ability of corticosteroids to reactivate $C$. pneumoniae infection. These data suggest that $C$. pneumoniae infection may influence the clinical course of asthma.

In the same way, preliminary data on chronic infection with C. pneumoniae in patients with COPD and its interaction with host cells indicate that this agent may be implicated in the modulation of the natural history of chronic bronchitis and emphysema [12].

The interesting finding of the study by GIEFFERs et al. [5] is the demonstration that granulocytes are one of the main target of $C$. pneumoniae infection and that these cells can act as "infecting" cells and reservoir of the pathogen. These data confirm the excellent capacity of $C$. pneumoniae to survive in different immune cells, use the immune cells as carriers for breaching the blood-tissue barriers, and potentially cause chronic/persistent infections.
Chronic infection is probably the real challenge in Chlamydia pneumoniae infection and we certainly need new studies addressing the question whether Chlamydia pneumoniae long-term survival within immune and nonimmune cells has a role in chronic pulmonary and extrapulmonary diseases.

\section{References}

1. Neuman FJ. Chlamydia pneumoniae-atherosclerosis link: a sound concept in search for clinical relevance. Circulation 2002; 106: 2414-2416.

2. Grimaldi LM, Pincherle A, Martinelli-Boneschi F, et al. An MRI study of Chlamydia pneumoniae infection in Italian multiple sclerosis patients. Mult Scler 2003; 9: 467-471.

3. Blain BJ, Gerard HC, Arking EJ, et al. Identification and localization of Chlamydia pneumoniae in the Alzheimer's brain. Med Microbiol Immunol 1998; 187: 23-42.

4. Dechend R, Maass M, Gieffer J, et al. Chlamydia pneumoniae infection of vascular smooth muscle and endothelial cells activates NF- $\kappa \mathrm{B}$ and induces tissue factor and PAI-1 expression: a potential link to accelerated arteriosclerosis. Circulation 1999; 100: 1369-1373.

5. Gieffers J, van Zandbergen G, Rupp J, et al. Phagocytes transmit Chlamydia pneumoniae from the lung to the vasculature. Eur Respir J 2004; 23: 506-510.

6. Wark PAB, Johnston SL, Simpson JL, Hensley MJ, Gibson PG. Chlamydia pneumoniae immunoglobulin A reactivation and airway inflammation in acute asthma. Eur Respir J 2002; 20: 834-840.

7. Moazed TC, Kuo CC, Grayston JT, Campbell LA. Evidence of systemic dissemination of Chlamydia pneumoniae via macrophages in the mouse. J Infect Dis 1998; 177: 13221325.

8. Blasi F, Boman J, Esposito G, et al. Chlamydia pneumoniae DNA detection in peripheral blood mononuclear cells is predictive of vascular infection. $J$ Infect Dis 1999; 180: 2074 2076.

9. May AE, Redecke V, Grüner S, et al. Recruitment of Chlamydia pneumoniae-infected macrophages to the carotid artery wall in noninfected, nonatherosclerotic mice. Arterioscler Thromb Vasc Biol 2003; 23: 789-794.

10. MacIntyre A, Abramov R, Hammond CJ, et al. Chlamydia pneumoniae infection promotes the transmigration of monocytes through human brain endothelial cells. J Neurosci Res 2003; 71: 740-750.

11. Lenz DC, Lu L, Conant SB, et al. A Chlamydia pneumoniaespecific peptide induces experimental autoimmune encephalomyelitis in rats. J Immunol 2001; 167: 1803-1808.

12. Blasi F, Damato S, Cosentini R, et al. Chlamydia pneumoniae and chronic bronchitis: association with severity and bacterial clearance following treatment. Thorax 2002; 57: 672-676.

13. Stephens RS. The cellular paradigm of chlamydial pathogenesis. TRENDS Microbiol 2003; 11: 44-51.

14. Cunningham AF, Johnston SL, Julious SA, Lampe FC, Ward ME. Chronic Chlamydia pneumoniae infection and asthma exacerbations in children. Eur Respir J 1998; 11: 345349.

15. Thumerelle C, Deschildre A, Bouquillon C, et al. Role of viruses and atypical bacteria in exacerbations of asthma in hospitalized children: a prospective study in the Nord-Pas de Calais region (France). Pediatr Pulmonol 2003; 35: 75-82.

16. ten Brinke A, van Dissel JT, Sterk JT, Zwinderman AH, Rabe KF, Bel EH. Persistent airflow limitation in adultonset nonatopic asthma is associated with serologic evidence of Chlamydia pneumoniae infections. J Allergy Clin Immunol 2001; 107: 449-454. 